\title{
World Development Report 2018 « Learning to Realize
} Education's Promise. Overview "

Banque mondiale, 2018, 52 p. [https://goo.gl/v3rcg8]

\section{Roger-François Gauthier}

\section{OpenEdition}

\section{Journals}

Édition électronique

URL : https://journals.openedition.org/ries/6107

DOI : 10.4000/ries.6107

ISSN : 2261-4265

Éditeur

France Education international

Édition imprimée

Date de publication : 30 avril 2018

Pagination : 27-29

ISBN : 978-2-85420-618-0

ISSN : 1254-4590

\section{Référence électronique}

Roger-François Gauthier, « World Development Report 2018 « Learning to Realize Education's Promise. Overview » », Revue internationale d'éducation de Sèvres [En ligne], 77 | avril 2018, mis en ligne le 30 avril 2018, consulté le 25 juin 2021. URL : http://journals.openedition.org/ries/6107 ; DOI : https://doi.org/ $10.4000 /$ ries.6107

Ce document a été généré automatiquement le 25 juin 2021

(c) Tous droits réservés 


\title{
World Development Report 2018 "Learning to Realize Education's Promise. Overview»
}

\author{
Banque mondiale, 2018, 52 p. [https://goo.gl/v3rcg8]
}

Roger-François Gauthier

1 Ce type de publication, sous une telle bannière, trouve en général toutes les ressources pour se faire connaître. Il est en revanche sans doute indispensable d'en connaître les grandes lignes et de se doter d'éléments de réflexion critique, car on sait qu'il va circuler, être lu dans le monde entier et qu'il risque de devenir le bréviaire de quantité d'acteurs nationaux ou internationaux, en imposant une rhétorique.

2 Il est certes symptomatique que la Banque mondiale consacre son rapport à l'éducation et qu'elle reprenne au fond ce qui se dit depuis une vingtaine d'années, à savoir que l'objectif du développement de l'éducation n'est pas la procédure de scolarisation mais bel et bien le fait que des élèves apprennent. Après tout, ni dans les pays développés ni les pays en développement, cela n'a été, sur cinquante ans, si évident qu'il ne soit pas toujours important de le rappeler : la scolarisation des élèves africains ne s'est pas faite avec moins de considération des apprentissages effectifs que la massification de l'enseignement secondaire français.

L'ouvrage avance de façon assurée, et il n'est pas sans pertinence de souligner au moins deux aspects d'une rhétorique fort travaillée.

La rhétorique née de la présentation de ce type d'ouvrage, d'abord, où le "réel » est censé être convoqué par la multiplication des tableaux, des chiffres et des encarts. L'idée est double : la multiplication de la description des pratiques («practices») est vertueuse en ce qu'elle crée une sorte d'espace mondial d'échange, de supermarché des bonnes idées pédagogiques, qui pourraient s'appliquer à Oslo, Dakar ou Yangon. Mais aussi toutes les activités relatives auxapprentissages ou aux politiques d'éducation peuvent être représentées par des données chiffrées, en outre facilement prédictives. 
5 La rhétorique même des idées de l'ouvrage est efficace. Une première partie détaille la " crise de l'apprentissage ", son objectif étant de faire apparaître la gravité sans recours d'une situation: les élèves apprennent mal ; certains, même, n'apprennent pas car ils ne sont pas scolarisés ; les pauvres (élèves ou nations) apprennent encore plus mal que les autres. Les deux étapes suivantes de l'analyse de la crise sont intéressantes: si les élèves apprennent mal, c'est que les écoles les desservent et si les écoles les desservent, c'est que les systèmes eux-mêmes ne font pas ce qu'il faudrait. C'est habile, implacable, et surtout aucun lecteur ne peut se sentir échapper à la noirceur du tableau. Comme chez Pascal, pourrait-on dire, la proposition de salut suit de peu le désespoir de l'analyse. Et ne cachons pas l'intérêt de ces pages : voir ainsi mis en cause, à propos de l'inefficacité des systèmes, les intérêts des politiciens, des technocrates, des éditeurs et des corporatismes divers est stimulant. On regrettera peut-être seulement que les responsabilités des organisations internationales diverses, dont la Banque mondiale, ne soient pas évoquées.

6 Ce salut est, lui aussi, remarquablement cadré dans sa présentation, puisqu'il réside dans trois perspectives. Passant de la trinité du désespoir à celle du salut, on découvre alors qu'elle réside dans la nécessité de mesurer les apprentissages, puis dans celle d'agir selon les preuves (avec des remarques intéressantes comme l'intérêt de repérer les écarts entre ce qu'on fait et ce qu'on pourrait faire, ainsi qu'un passage bienvenu sur les "principes ", hélas jargonnant, le seul dans ce cas), enfin d'agir en mettant en cohérence les différents intérêts qui tirent à hue et à dia.

7 Curieusement, ce livre, qui contraint par tant d'habileté le lecteur à adhérer à ses thèses générales, est dans le détail beaucoup moins sûr de lui : dans la jungle d'un grand nombre d'encarts ("boxes»), tout est à peu près dit et son contraire, ce qui facilité l'éloge devant la prudence des auteurs (on lit par exemple avec étonnement qu'il n'y a pas de solutions à l'échelle mondiale, «there are no global solutions ») et rend délicate la critique.

8 Critique que nous construirons pourtant dans quatre directions :

1. alors que le texte semble, d'une part, ambitieux sur l'ensemble des pratiques qui, d'une façon ou d'une autre portent le nom d'« évaluation", et d'autre part volontaire pour " aligner » les différents aspects d'une politique, on peut regretter sa timidité à « aligner » les différentes hypostases de l'évaluation, et, par exemple, de ne pas citer à comparaître les systèmes d'examens, qui détricotent si souvent, pourtant, les intentions du curriculum ;

2. une vision étriquée de la publication des soi-disant performances des écoles, sans réflexion critique sur les effets souvent pervers de ce type de pratique, sans proposer de logique plus intelligente d'évaluation du contexte d'action de chaque école et de ses objectifs, qui peuvent être spécifiques ;

3. une confirmation de cette vision restreinte de la performance des élèves : on décrit l'enfance et les apprentissages sur le mode de « rattrapages » permanents, de retards à combler. Tout cela va jusqu'à des présentations où la manie du chiffre n'empêche pas, comme souvent, l'insuffisance intellectuelle aussi bien qu'éthique du propos : que signifie, et comment peuton oser écrire qu'il faudra 180 ans aux élèves tunisiens pour, en mathématiques et en moyenne, rejoindre le niveau moyen de leurs camarades de l'OCDE ?

4. L'absence à peu près totale d'interrogation sur les contenus des apprentissages («Learning What?»). On ne parle que d'un certain type de savoirs, élémentaires, ceux qui sont valorisés dans la vulgate mondiale, en faisant comme si ces savoirs allaient conduire en tous les cas chacun aux compétences de réflexion de haut niveau « High Level Thinking Skills ». Apprendre quoi? Ne pas considérer cette question comme incontournable, c'est faire comme si les 
écoles du monde, conduisaient tous les enfants à l'émancipation et à assumer le plus d'humanité. Il n'existe pas dans le monde, bien sûr, d'écoles obéissant à des tyrans ? d'écoles insufflant aux élèves divers racismes ou sentiments d'exclusion? diverses haines ?, d'écoles obéissant à une obsession de compétition, qui rend l'enfance ici ou là insupportable?

\section{AUTEUR}

\section{ROGER-FRANÇOIS GAUTHIER}

Roger-François Gauthier, inspecteur général de l'administration de l'éducation nationale et de la recherche, est membre du Conseil supérieur des programmes. Ses travaux, au sein des institutions françaises et comme chercheur ou consultant auprès de plusieurs organisations internationales (Unesco, Organisation internationale de la Francophonie portent notamment sur les contenus d'enseignement et les politiques curriculaires, sujets à propos desquels il a écrit plusieurs ouvrages. Courriel : roger-francois.gauthier@education.gouv.fr 